\title{
A Nonmonotone Hybrid Method of Conjugate Gradient and Lanczos-type for Solving Nonlinear Systems
}

\author{
Chun-Xia Jia $\cdot$ Jue-Yu Wang $\cdot$ De-Tong Zhu
}

Received: 8 October 2013/Revised: 22 July 2014/ Accepted: 26 July 2014/

Published online: 2 September 2014

(C) Operations Research Society of China, Periodicals Agency of Shanghai University, and SpringerVerlag Berlin Heidelberg 2014

\begin{abstract}
In this paper, we construct a new algorithm which combines the conjugate gradient and Lanczos methods for solving nonlinear systems. The iterative direction can be obtained by solving a quadratic model via conjugate gradient and Lanczos methods. Using the backtracking line search, we will find an acceptable trial step size along this direction which makes the objective function nonmonotonically decreasing and makes the norm of the step size monotonically increasing. Global convergence and local superlinear convergence rate of the proposed algorithm are established under some reasonable conditions. Finally, we present some numerical results to illustrate the effectiveness of the proposed algorithm.
\end{abstract}

Keywords Nonmonotonic technique - Nonlinear systems · Lanczos method · Conjugate gradient

The authors gratefully acknowledge the partial supports of the National Natural Science Foundation of China (No. 11371253).

C.-X. Jia $(\bowtie) \cdot$ J.-Y. Wang

Department of Mathematics, Shanghai Normal University, Shanghai 200234, China

e-mail: jiachx@shnu.edu.cn

J.-Y. Wang

e-mail: shnu201005@hotmail.com

D.-T. Zhu

Department of Mathematics, Shanghai Normal University, Guilin Road 100, Shanghai 200234,

China

e-mail: dtzhu@shnu.edu.cn 


\section{Introduction}

This paper is concerned with the development of conjugate gradient and Lanczos methods for the solution of nonlinear systems:

$$
F(x)=0,
$$

where $F: \mathbb{R}^{n} \rightarrow \mathbb{R}^{n}$ is a given continuously differentiable mapping.

There are quite a few literatures proposing affine-scaling algorithm for solving problems appeared during the last few years. Sun [15] gave a convergence proof for an affine-scaling algorithm for convex quadratic programming without nondegeneracy assumptions, and Ye [17] introduced affine-scaling algorithm for nonconvex quadratic programming. Classical methods also can be used to solve (1.1), for example, nonlinear conjugate gradient method, which can be easily programmed and computed, is one of the most popular and useful method for solving large-scale optimization problems(see $[3,4,9,10])$. The idea of conjugate gradient path in unconstrained optimization is given in [1]; which is defined as linear combination of a sequence of conjugate directions that are obtained by applying standard conjugate direction method to approximate quadratic function of unconstrained optimization. The Lanczos method for solving the quadratic-model trust region subproblem in a weighted $l_{2}$-norm is proposed by Gould et al. in [5]. By combining Lanczos method with conjugate gradient path, we can construct a new path (see [7, 11]), which has both properties of Lanczos vectors and properties of conjugate gradient path.

Stimulated by the progress in these aspects, in this paper, an algorithm via the conjugate gradient and Lanczos methods is proposed to solve (1.1). Define the merit function

$$
f(x)=\frac{1}{2}\|F(x)\|^{2}=\frac{1}{2} \sum_{i=1}^{n} F_{i}^{2}(x) .
$$

The necessary condition of the problem (1.1) is to solve the following optimization problem $\min f(x)$. The basic idea in the proposed algorithm is based on the minimal value of the following quadratic programming subproblem

$$
\min \psi_{k}(p)=\frac{1}{2}\left\|F_{k}^{\prime} p+F_{k}\right\|^{2}=\frac{1}{2} p^{\mathrm{T}} F_{k}^{\prime \mathrm{T}} F_{k}^{\prime} p+g_{k}^{\mathrm{T}} p+\frac{1}{2}\left\|F_{k}\right\|^{2}
$$

where $F_{k}=F\left(x_{k}\right), F_{k}^{\prime}=F^{\prime}\left(x_{k}\right), g_{k}=\nabla f\left(x_{k}\right)=F_{k}^{\prime} \mathrm{T} F_{k}$, and $\psi_{k}(p)$ is an adequate representation of $f(x)$ around $x_{k}$.

The paper is organized as follows. In Sect. 2, the concrete algorithm for solving (1.1) is stated. In Sect. 3, we prove the global convergence of the proposed algorithm. Further, we establish that the proposed algorithm has strong global convergence and local convergence rate in Sect. 4. Finally, the results of numerical experiments of the proposed algorithm are reported in Sect. 5. 


\section{Algorithm}

This section describes and designs the conjugate gradient and Lanczos methods in association with nonmonotonic backtracking technique for solving the nonlinear system (1.1).

\subsection{Algorithm NCGL}

We are now in a position to give a precise statement of the nonmonotone hybrid method of conjugate gradient and lanczos technique.

\section{Initialization Step}

Choose parameters $\beta \in\left(0, \frac{1}{2}\right), \omega \in(0,1), \varepsilon>0$ and positive integer $M$ as nonmonotonic parameter. Let $m(0)=0$ and $\xi \in(0,1)$, give a starting strict feasibility interior point $x_{0} \in \mathbb{R}^{n}$. Set $k=0$, go to the main step.

\section{Main Step}

1. Evaluate $f_{k}=f\left(x_{k}\right) \stackrel{\text { def }}{=} \frac{1}{2}\left\|F\left(x_{k}\right)\right\|^{2}, g_{k}=\nabla f\left(x_{k}\right) \stackrel{\text { def }}{=}\left(F_{k}^{\prime}\right)^{\mathrm{T}} F_{k}$.

2. If $\left\|g_{k}\right\|=\left\|\left(F_{k}^{\prime}\right)^{\mathrm{T}} F_{k}\right\| \leqslant \varepsilon$, stop with the approximate solution $x_{k}$.

3. $q_{0}=0, v_{1}=0, r_{1}=\nabla$

$\psi_{k}\left(v_{1}\right)=g_{k}, d_{1}=-g_{k}, \theta_{1}=1, \gamma_{1}=\left\|r_{1}\right\|, q_{1}=\frac{r_{1}}{\gamma_{1}}$. Let $i=1$.

4. Compute $w_{i}=F_{k}^{\prime} d_{i}$. If

$$
\begin{gathered}
\left\|w_{i}\right\| \neq 0 \\
r_{i} \neq 0
\end{gathered}
$$

go to step 5, otherwise go to step 6 .

5. Calculate

$$
\begin{aligned}
\lambda_{i} & =\frac{\theta_{i}^{2}\left\|r_{i}\right\|^{2}}{\left\|w_{i}\right\|^{2}}, \\
v_{i+1} & =v_{i}+\lambda_{i} d_{i}, \\
\theta_{i+1} & =-\lambda_{i} \theta_{i} \gamma_{i}, \\
\delta_{i} & =\left\|F_{k}^{\prime} q_{i}\right\|^{2}, \\
r_{i+1} & =F_{k}^{\prime \mathrm{T}} F_{k}^{\prime} q_{i}-\delta_{i} q_{i}-\gamma_{i} q_{i-1}, \\
\gamma_{i+1} & =\left\|r_{i+1}\right\|, \\
q_{i+1} & =\frac{r_{i+1}}{\gamma_{i+1}}, \\
\beta_{i} & =\frac{\theta_{i+1} r_{i+1}^{\mathrm{T}} F_{k}^{\prime}{ }^{\prime} w_{i}}{\left\|w_{i}\right\|^{2}}, \\
d_{i+1} & =-\theta_{i+1} r_{i+1}+\beta_{i} d_{i} .
\end{aligned}
$$


Calculate

$$
f\left(x_{k}\right)-f\left(x_{k}+v_{i+1}\right) \geqslant \xi\left[f\left(x_{k}\right)-\psi_{k}\left(v_{i+1}\right)\right] .
$$

If (2.3) is not satisfied, set $i \Leftarrow i+1$, go to 4 .

6. If $i=1, p_{k}=d_{1}$, otherwise, $p_{k}=v_{i}$.

7. Choose $\alpha_{k}=1, \omega, \omega^{2}, \cdots$, until the following inequality is satisfied:

$$
f\left(x_{k}+\alpha_{k} p_{k}\right) \leqslant f\left(x_{l(k)}\right)+\alpha_{k} \beta g_{k}^{\mathrm{T}} p_{k},
$$

where $f\left(x_{l(k)}\right)=\max _{0 \leqslant j \leqslant m(k)}\left\{f\left(x_{k-j}\right)\right\}$.

8. Set

$$
x_{k+1}=x_{k}+\alpha_{k} p_{k} \text {. }
$$

9. Take the nonmonotone control parameter $m(k+1)=\min \{m(k)+1, M\}$. Then set $k \leftarrow k+1$ and go to step 1 .

\subsection{Properties of the Proposed Algorithm}

The following lemmas give some properties of the algorithm.

Lemma 2.1 (see $[7,11])$ Suppose that the directions $q_{i}$ and $d_{i}$ are generated by step 5 of Algorithm NCGL, $1 \leqslant i \leqslant l \leqslant n_{k}$, the following properties hold:

$$
\begin{gathered}
q_{i}^{\mathrm{T}} q_{j}=0, \quad 1 \leqslant j<i \leqslant l \leqslant n_{k} \\
Q_{i}^{\mathrm{T}} F_{k}^{\prime \mathrm{T}} F_{k}^{\prime} Q_{i}=T_{i}, \quad i=1,2, \cdots, n_{k} \\
r_{i}^{\mathrm{T}} d_{j}=0, \quad 1 \leqslant j<i \leqslant l \leqslant n_{k} \\
w_{i}^{\mathrm{T}} w_{j}=0, \quad i \neq j \\
d_{i}^{\mathrm{T}} d_{j} \geqslant 0, \quad 1 \leqslant i, j \leqslant n_{k}
\end{gathered}
$$

where $Q_{i}=\left[q_{1}, q_{2}, \cdots, q_{i}\right]$ and the tridiagonal matrix $T_{i}$ is

$$
T_{i}=\left[\begin{array}{ccccc}
\delta_{1} & \gamma_{2} & & & \\
\gamma_{2} & \delta_{2} & \gamma_{3} & & \\
& \ddots & \ddots & \ddots & \\
& & & \delta_{i-1} & \gamma_{i} \\
& & & \gamma_{i} & \delta_{i}
\end{array}\right] .
$$


Lemma 2.2 (see $[7,11])$ Suppose that $\nabla \psi_{k}\left(v_{i+1}\right)=F_{k}^{\prime \mathrm{T}} F_{k}^{\mathrm{T}} v_{i+1}+g_{k}=\theta_{i+1} r_{i+1}$ (see [ 5]), where $\theta_{i+1}=\left\langle e_{i+1}, h_{i+1}\right\rangle, e_{i}$ is a unit vector and its the ith $1, h_{i+1}$ satisfy $T_{i+1} h_{i+1}+\gamma_{1} e_{1}=0$. Then we have

$$
\theta_{i+1}=-\lambda_{i} \theta_{i} \gamma_{i}\left(\theta_{1}=1\right)
$$

\section{Global Convergence Analysis}

Throughout this section, we assume that $F: \mathbb{R}^{n} \rightarrow \mathbb{R}^{n}$ is continuously differentiable. Given $x_{0} \in \mathbb{R}^{n}$, the algorithm generates a sequence $\left\{x_{k}\right\} \subset \mathbb{R}^{n}$. In our analysis, the level set of $f$ is denoted by

$$
\mathcal{L}\left(x_{0}\right)=\left\{x \in \mathbb{R}^{n} \mid f(x) \leqslant f\left(x_{0}\right)\right\} .
$$

In order to discuss the properties of Algorithm NCGL in detail, we will summarize as follows.

Lemma 3.1 Consider the step $v_{j}$ be obtained from NCGL. Then the norm of the step $v_{j}$ is monotonically increasing and the quadratic function $\psi_{k}\left(v_{j}\right)$ is monotonically decreasing, that is, $\left\|v_{j}\right\| \leqslant\left\|v_{j+1}\right\|$ and $\psi_{k}\left(v_{j+1}\right) \leqslant \psi_{k}\left(v_{j}\right)$.

Proof Because $v_{1}=0, \lambda_{i}>0$ and $v_{j}^{\mathrm{T}} d_{j}=\sum_{i=1}^{j-1} \lambda_{i} d_{i}^{\mathrm{T}} d_{j} \geqslant 0$,

$$
\begin{aligned}
\left\|v_{j+1}\right\|^{2} & =\left(v_{j}+\lambda_{j} d_{j}\right)^{\mathrm{T}}\left(v_{j}+\lambda_{j} d_{j}\right) \\
& =\left\|v_{j}\right\|^{2}+2 \lambda_{j} v_{j}^{\mathrm{T}} d_{j}+\lambda_{j}^{2}\left\|d_{j}\right\|^{2} \geqslant\left\|v_{j}\right\|^{2},
\end{aligned}
$$

which means that $\left\|v_{j}\right\| \leqslant\left\|v_{j+1}\right\|$ holds.

Using the expression of $\psi_{k}, v_{j}$ and (2.9), it is clear that

$$
\begin{aligned}
& \psi_{k}\left(v_{j+1}\right)-\psi_{k}\left(v_{j}\right) \\
& =g_{k}^{\mathrm{T}}\left(v_{j+1}-v_{j}\right)+\frac{1}{2} v_{j+1}^{\mathrm{T}} F_{k}^{\prime \mathrm{T}} F_{k}^{\prime} v_{j+1}-\frac{1}{2} v_{j}^{\mathrm{T}} F_{k}^{\prime \mathrm{T}} F_{k}^{\prime} v_{j} \\
& =\lambda_{j} g_{k}^{\mathrm{T}} d_{j}+\frac{1}{2}\left(\sum_{i=1}^{j} \lambda_{i} d_{i}\right)^{\mathrm{T}} F_{k}^{\prime \mathrm{T}} F_{k}^{\prime}\left(\sum_{i=1}^{j} \lambda_{i} d_{i}\right)-\frac{1}{2}\left(\sum_{i=1}^{j-1} \lambda_{i} d_{i}\right)^{\mathrm{T}} F_{k}^{\prime \mathrm{T}} F_{k}^{\prime}\left(\sum_{i=1}^{j-1} \lambda_{i} d_{i}\right) \\
& =\lambda_{j} g_{k}^{\mathrm{T}} d_{j}+\frac{1}{2} \lambda_{j}^{2}\left\|w_{j}\right\|^{2} \\
& =\frac{1}{2} \lambda_{j}\left[2 g_{k}^{\mathrm{T}} d_{j}+\theta_{j}^{2} r_{j}^{\mathrm{T}} r_{j}\right] .
\end{aligned}
$$


Noting

$$
\begin{aligned}
g_{k}^{\mathrm{T}} d_{j}+\theta_{j}^{2} r_{j}^{\mathrm{T}} r_{j} & =d_{j}^{\mathrm{T}} r_{1}-\theta_{j} r_{j}^{\mathrm{T}}\left(-\theta_{j} r_{j}+\beta_{j-1} d_{j-1}\right)=d_{j}^{\mathrm{T}} r_{1}-\theta_{j} r_{j}^{\mathrm{T}} d_{j} \\
& =d_{j}^{\mathrm{T}}\left(r_{1}-\theta_{j} r_{j}\right)=d_{j}^{\mathrm{T}}\left(r_{1}-g_{k}-F_{k}^{\prime \mathrm{T}} F_{k}^{\prime} v_{j}\right)=-d_{j}^{\mathrm{T}} \sum_{i=1}^{j-1} \lambda_{i} F_{k}^{\prime \mathrm{T}} F_{k}^{\prime} d_{i}=0
\end{aligned}
$$

and $\theta_{j}^{2} r_{j}^{\mathrm{T}} r_{j} \geqslant 0$, we $\quad$ get $\quad g_{k}^{\mathrm{T}} d_{j} \leqslant 0, \quad$ so $2 g_{k}^{\mathrm{T}} d_{j}+\theta_{j}^{2} r_{j}^{\mathrm{T}} r_{j}<0$, that is, $\psi_{k}\left(v_{j+1}\right)-\psi_{k}\left(v_{j}\right) \leqslant 0$. This completes the proof of this lemma.

The following lemma shows the relation between the gradient $g_{k}$ of the objective function and the step $p_{k}$ generated by the proposed algorithm. We can see from the following lemma that the direction of the trial step is a sufficiently descent direction.

Assumption 3.2 Sequence $\left\{x_{k}\right\}$ generated by the algorithm is contained in the compact set $\mathcal{L}\left(x_{0}\right)$.

Assumption 3.3 $\left\|p_{k}\right\|$ and $F_{k}^{\prime} F_{k}^{\prime}$ are uniformly bounded, that is, there exist constants $\chi_{p}$ and $\chi$ satisfy $\left\|p_{k}\right\| \leqslant \chi_{p}$ and $\left\|F_{k}^{\prime} \mathrm{T} F_{k}^{\prime}\right\| \leqslant \chi$ for all $k$.

Lemma 3.4 Consider the step $p_{k}=v_{j}$ be obtained from NCGL. Then

(1) $\left\{g_{k}^{\mathrm{T}} v_{j}\right\}$ is monotonically decreasing, that is, $g_{k}^{\mathrm{T}} v_{j+1} \leqslant g_{k}^{\mathrm{T}} v_{j}, 1 \leqslant j \leqslant n_{k}$.

(2) $g_{k}^{\mathrm{T}} p_{k}$ satisfies the following sufficient descent condition

$$
g_{k}^{\mathrm{T}} p_{k} \leqslant-\min \left\{1, \frac{1}{\chi}\right\}\left\|g_{k}\right\|^{2} .
$$

Proof

(1) From (2.10), the following is true:

$$
g_{k}^{\mathrm{T}} v_{j+1}-g_{k}^{\mathrm{T}} v_{j}=g_{k}^{\mathrm{T}}\left(v_{j+1}-v_{j}\right)=\lambda_{j} g_{k}^{\mathrm{T}} d_{j}=-\lambda_{j} d_{1}^{\mathrm{T}} d_{j} \leqslant 0 .
$$

(2) If $\left\|w_{1}\right\|=0$, then $p_{k}=v_{1}=d_{1}$ and

$$
g_{k}^{\mathrm{T}} p_{k}=g_{k}^{\mathrm{T}} d_{1}=-g_{k}^{\mathrm{T}} g_{k}=-\left\|g_{k}\right\|^{2} \leqslant-C_{1}\left\|g_{k}\right\|^{2} .
$$

If $\left\|w_{1}\right\|>0$, then there exists $j_{0} \geqslant 2$ such that $p_{k}=v_{j_{0}}$. The results that $\left\{g_{k}^{\mathrm{T}} v_{j}\right\}$ is monotonically decreasing and $g_{k}^{\mathrm{T}} v_{2}=g_{k}^{\mathrm{T}}\left(v_{1}+\lambda_{1} d_{1}\right)=\lambda_{1} g_{k}^{\mathrm{T}} d_{1}=-\lambda_{1}\left\|g_{k}\right\|^{2}$ yield:

$$
g_{k}^{\mathrm{T}} p_{k} \leqslant g_{k}^{\mathrm{T}} v_{2}=-\lambda_{1}\left\|g_{k}\right\|^{2} .
$$


Assumption 3.3 shows $\lambda_{1}=\frac{\theta_{1}^{2}\left\|r_{1}\right\|^{2}}{d_{1}^{\mathrm{T}} F_{k}^{\mathrm{T}} F_{k}^{\prime} d_{1}} \geqslant \frac{\left\|g_{k}\right\|^{2}}{\left\|g_{k}\right\|^{2} \cdot\left\|F_{k}^{\prime T} F_{k}^{\prime}\right\|} \geqslant \frac{1}{\chi}$. Therefore,

$$
g_{k}^{\mathrm{T}} p_{k} \leqslant-\frac{1}{\chi}\left\|g_{k}\right\|^{2} \leqslant-\min \left\{1, \frac{1}{\chi}\right\}\left\|g_{k}\right\|^{2} .
$$

Lemma 3.5 The predicted reduction satisfies the estimate:

$$
f\left(x_{k}\right)-\psi_{k}\left(p_{k}\right) \geqslant\left\|g_{k}\right\|^{2} \min \left\{1, \frac{1}{2 \chi}\right\} .
$$

Proof The proof is analogous to that of Lemma 3.4. If $\left\|w_{1}\right\|=0$, then $p_{k}=v_{1}=$ $d_{1}$ and

$$
f\left(x_{k}\right)-\psi_{k}\left(p_{k}\right)=-g_{k}^{\mathrm{T}} d_{1}-\frac{1}{2}\left\|w_{1}\right\|^{2}=-g_{k}^{\mathrm{T}} d_{1}=\left\|g_{k}\right\|^{2} .
$$

For the case of $\left\|w_{1}\right\|>0$, Since $\left\{\psi_{k}\left(v_{j}\right)\right\}$ is monotonically decreasing, it follows that

$$
\begin{aligned}
f\left(x_{k}\right)-\psi_{k}\left(p_{k}\right) & \geqslant f\left(x_{k}\right)-\psi_{k}\left(\lambda_{1} d_{1}\right) \\
& =-\lambda_{1} g_{k}^{\mathrm{T}} d_{1}-\frac{1}{2} \lambda_{1}^{2}\left\|w_{1}\right\|^{2}=\lambda_{1}\left\|g_{k}\right\|^{2}-\frac{\lambda_{1}}{2}\left\|g_{k}\right\|^{2} \\
& =\frac{\lambda_{1}}{2}\left\|g_{k}\right\|^{2} \geqslant\left\|g_{k}\right\|^{2} \min \left\{1, \frac{1}{2 \chi}\right\} .
\end{aligned}
$$

The conclusion of the lemma holds.

We are now ready to state one of our main results of the proposed algorithm, which also needs the following assumptions.

Assumption 3.6 $g(x)=\nabla f(x)$ is Lipschitz continuous, that is, there exists a constant $\gamma$ such that

$$
\|g(x)-g(y)\| \leqslant \gamma\|x-y\| \forall x, y \in \mathcal{L}\left(x_{0}\right) .
$$

Assumption 3.7 $F_{*}^{\prime}=F^{\prime}\left(x_{*}\right)$ is nonsingular, where $x_{*}$ is the limit point.

Theorem 3.8 Assume that Assumptions 3.2, 3.3 and 3.6 hold. Let $\left\{x_{k}\right\} \subset \mathbb{R}^{n}$ be a sequence generated by NCGL. Then

$$
\liminf _{k \rightarrow \infty}\left\|F_{k}^{\prime T} F_{k}\right\|=0 .
$$

Proof Taking into account that $m(k+1) \leqslant m(k)+1$ and $f\left(x_{k+1}\right) \leqslant f\left(x_{l}(k)\right)$, we get

$$
f\left(x_{l(k+1)}\right)=\max _{0 \leqslant j \leqslant m(k+1)} f\left(x_{k+1-j}\right) \leqslant \max _{0 \leqslant j \leqslant m(k)+1} f\left(x_{k+1-j}\right)=f\left(x_{l(k)}\right) .
$$

This means $\left\{f\left(x_{l(k)}\right)\right\}$ is nonincreasing for all $k$ and hence $\left\{f\left(x_{l(k)}\right)\right\}$ is convergent. If the conclusion of the theorem is not true, there exists some $\varepsilon>0$ such that 


$$
\left\|F_{k}^{\prime \mathrm{T}} F_{k}\right\| \geqslant \varepsilon .
$$

From (2.4) and (3.1), we obtain

$$
\begin{aligned}
f\left(x_{l(k)}\right) & =f\left(x_{l(k)-1}+\alpha_{l(k)-1} p_{l(k)-1}\right) \\
& \leqslant f\left(x_{l(l(k)-1)}\right)+\beta \alpha_{l(k)-1} g_{l(k)-1}^{\mathrm{T}} p_{l(k)-1} \\
& \leqslant f\left(x_{l(l(k)-1)}\right)-\alpha_{l(k)-1} \beta \varepsilon^{2} \min \left\{1, \frac{1}{\chi}\right\} .
\end{aligned}
$$

Since $\left\{f\left(x_{l(k)}\right)\right\}$ is convergent, it follows from (3.4) that

$$
\lim _{k \rightarrow \infty} \alpha_{l(k)-1}=0
$$

Equation (3.5) and Assumption 3.3 imply $\lim _{k \rightarrow \infty} \alpha_{l(k)-1}\left\|p_{l(k)-1}\right\|=0$. Analogous to the proof of theorem in [6], we have

$$
\lim _{k \rightarrow \infty} f\left(x_{k}\right)=\lim _{k \rightarrow \infty} f\left(x_{l(k)}\right) .
$$

Similar to the proof of (3.5), we obtain

$$
\lim _{k \rightarrow \infty} \alpha_{k}=0 .
$$

The acceptance rule in step 7 yields

$$
f\left(x_{k}+\frac{\alpha_{k}}{\omega} p_{k}\right)>f\left(x_{l(k)}\right)+\frac{\alpha_{k}}{\omega} \beta g_{k}^{\mathrm{T}} p_{k} \geqslant f\left(x_{k}\right)+\frac{\alpha_{k}}{\omega} \beta g_{k}^{\mathrm{T}} p_{k},
$$

On the other hand, by Taylor's Theorem and Assumption 3.6,

$$
\begin{aligned}
f\left(x_{k}+\frac{\alpha_{k}}{\omega} p_{k}\right)-f\left(x_{k}\right) & =\frac{\alpha_{k}}{\omega} g_{k}^{\mathrm{T}} p_{k}+\frac{\alpha_{k}}{\omega} \int_{0}^{1} g\left(x_{k}+t \frac{\alpha_{k}}{\omega} p_{k}-g\left(x_{k}\right)\right)^{\mathrm{T}} p_{k} \mathrm{~d} t \\
& \leqslant \frac{\alpha_{k}}{\omega} g_{k}^{\mathrm{T}} p_{k}+\frac{1}{2} \gamma\left(\frac{\alpha_{k}}{\omega}\right)^{2}\left\|p_{k}\right\|^{2}
\end{aligned}
$$

where $\gamma$ is Lipschitz constant for $g(x)$. From (3.7) and (3.8), we have

$$
\frac{\alpha_{k}}{\omega} g_{k}^{\mathrm{T}} p_{k}+\frac{1}{2} \gamma\left(\frac{\alpha_{k}}{\omega}\right)^{2}\left\|p_{k}\right\|^{2}>\beta \frac{\alpha_{k}}{\omega} g_{k}^{\mathrm{T}} p_{k} .
$$

So

$$
\alpha_{k} \geqslant \frac{2 \omega(\beta-1)}{\gamma\left\|p_{k}\right\|^{2}} g_{k}^{\mathrm{T}} p_{k} \geqslant \frac{2 \omega(1-\beta)}{\gamma \chi_{p}^{2}} \min \left\{1, \frac{1}{\chi}\right\} \varepsilon^{2}>0 .
$$

The observation that $\lim _{k \rightarrow \infty} \alpha_{k} \geqslant \frac{2 \omega(1-\beta)}{\gamma \chi_{p}^{2}} \min \left\{1, \frac{1}{\chi}\right\} \varepsilon^{2}>0$ contradicts (3.6). 


\section{Properties of the Local Convergence}

Theorem 3.8 indicates that at least one limit point of $\left\{x_{k}\right\}$ is a stationary point. In this section, we shall first extend this theorem to a stronger result and the local convergent rate.

Theorem 4.1 Assume that Assumptions 3.2, 3.3 and 3.6 hold. Let $\left\{x_{k}\right\}$ be a sequence generated by Algorithm NCGL. Then

$$
\lim _{k \rightarrow+\infty}\left\|F_{k}^{\prime \mathrm{T}} F_{k}\right\|=0
$$

Proof Assuming that the conclusion is not true, there is an $\varepsilon_{1} \in(0,1)$ and a subsequence $\left\{\left(F_{m_{i}}^{\prime}\right)^{\mathrm{T}} F_{m_{i}}\right\}$ such that for all $m_{i}, i=1,2, \cdots$

$$
\left\|\left(F_{m_{i}}^{\prime}\right)^{\mathrm{T}} F_{m_{i}}\right\| \geqslant \varepsilon_{1} \text {. }
$$

Consider any index $m_{i}$ such that $\left\|\nabla f_{m_{i}}\right\| \geqslant \varepsilon_{1}$. Assumption 3.6 implies

$$
\left\|\nabla f(x)-\nabla f\left(x_{m_{i}}\right)\right\| \leqslant \gamma\left\|x-x_{m_{i}}\right\| .
$$

Defining the scalar $R=\frac{n-1}{n r} \varepsilon_{1}$ and the ball $\mathcal{B}\left(x_{m_{i}}, R\right)=\left\{x \mid\left\|x-x_{m_{i}}\right\| \leqslant R\right\}$, where $n$ can be some very large integer. If $x \in \mathcal{B}\left(x_{m_{i}}, R\right)$, then

$$
\begin{aligned}
\|\nabla f(x)\| & \geqslant\left\|\nabla f_{m_{i}}\right\|-\left\|\nabla f(x)-\nabla f_{m_{i}}\right\| \\
& \geqslant \varepsilon_{1}-\gamma\left\|x-x_{m_{i}}\right\| \geqslant \varepsilon_{1}-\frac{n-1}{n} \varepsilon_{1}=\frac{1}{n} \varepsilon_{1}=\varepsilon_{2},
\end{aligned}
$$

where $\varepsilon_{2}=\frac{1}{n} \varepsilon_{1}$. If the entire sequence $\left\{x_{k}\right\}_{k \geqslant m_{i}}$ stays the ball $\mathcal{B}\left(x_{m_{i}}, R\right)$, we would have $\left\|\nabla f_{k}\right\| \geqslant \varepsilon_{2}>0$ for all $k \geqslant m_{i}$. The reasoning in the proof of Theorem 3.8 can be used to show that this scenario does not occur. Therefore, the sequence $\left\{x_{k}\right\}_{k \geqslant m_{i}}$ eventually leaves $\mathcal{B}\left(x_{m_{i}}, R\right)$, and there exists another subsequence $\left\{\left(F_{n_{i}}^{\prime}\right)^{\mathrm{T}} F_{n_{i}}\right\}$ such that

$$
\left\|\left(F_{k}^{\prime}\right)^{\mathrm{T}} F_{k}\right\| \geqslant \varepsilon_{2}, \text { for } m_{i} \leqslant k<n_{i}
$$

and

$$
\left\|\left(F_{n_{i}}^{\prime}\right)^{\mathrm{T}} F_{n_{i}}\right\| \leqslant \varepsilon_{2},
$$

for an $\varepsilon_{2} \in\left(0, \varepsilon_{1}\right)$.

Similar to the proof of Theorem 3.8, we have

$$
\lim _{k \rightarrow \infty, m_{i} \leqslant k<n_{i}} f\left(x_{l(k)}\right)=\lim _{k \rightarrow \infty, m_{i} \leqslant k<n_{i}} f\left(x_{k}\right) .
$$

The acceptance rule in step 7 yields

$$
f\left(x_{l(k)}\right)-f\left(x_{k}+\alpha_{k} p_{k}\right) \geqslant-\alpha_{k} \beta g_{k}^{\mathrm{T}} p_{k} \geqslant \alpha_{k} \beta \tau \varepsilon_{2} C_{1} \geqslant 0 .
$$

It follows from this that $\lim _{k \rightarrow \infty, m_{i} \leqslant k<n_{i}} \alpha_{k}=0$, which contradicts to (3.9). So (4.1) holds. 
The following theorem shows the convergence rate for the proposed algorithm.

Theorem 4.2 Assume that $F(x)$ is twice continuously differentiable, Assumptions 3.2, 3.3, 3.6 and 3.7 hold and $\left\{x_{k}\right\}$ is a sequence produced by Algorithm NCGL which convergence to $x_{*}$. Then the convergence is superlinear. i.e.,

$$
\lim _{k \rightarrow \infty} \frac{\left\|x_{k+1}-x_{*}\right\|}{\left\|x_{k}-x_{*}\right\|}=0
$$

Proof From Lemma 2.1,

$$
0=\theta_{j} r_{j}^{\mathrm{T}}\left(\sum_{i=1}^{j-1} \lambda_{i} d_{i}\right)=\theta_{j} r_{j}^{\mathrm{T}} v_{j}=\left(g_{k}+F_{k}^{\prime \mathrm{T}} F_{k}^{\prime} v_{j}\right)^{\mathrm{T}} v_{j}=g_{k}^{\mathrm{T}} v_{j}+v_{j}^{\mathrm{T}} F_{k}^{\prime \mathrm{T}} F_{k}^{\prime} v_{j} .
$$

Assumption 3.7 implies $F_{k}^{\prime} \mathrm{T} F_{k}^{\prime}$ is positive definite uniformly for sufficiently large $k$, so

$$
v_{j}^{\mathrm{T}} F_{k}^{\prime} \mathrm{T} F_{k}^{\prime} v_{j} \geqslant \zeta\left\|v_{j}\right\|^{2}
$$

where $\zeta>0$ is a constant. Equations (4.4) and (4.5) show

$$
\zeta\left\|v_{j}\right\|^{2} \leqslant v_{j}^{\mathrm{T}} F_{k}^{\prime} F_{k}^{\prime} v_{j}=-g_{k}^{\mathrm{T}} v_{j} \leqslant\left\|g_{k}\right\| \cdot\left\|v_{j}\right\| .
$$

It follows from Theorem 4.1 that

$$
\left\|v_{j}\right\| \leqslant \frac{1}{\zeta}\left\|g_{k}\right\| \rightarrow 0
$$

Noting $F(x)$ is twice continuously differentiable and $F\left(x_{*}\right)=0$, we have

$$
\begin{aligned}
& \left|\psi_{k}\left(v_{j}\right)-f\left(x_{k}+v_{j}\right)\right| \\
& =\left|g_{k}^{\mathrm{T}} v_{j}+\frac{1}{2} v_{j}^{\mathrm{T}} F_{k}^{\prime \mathrm{T}} F_{k}^{\prime} v_{j}-\left(g_{k}^{\mathrm{T}} v_{j}+\frac{1}{2} v_{j}^{\mathrm{T}} \nabla^{2} f\left(x_{k}\right) v_{j}+o\left(\left\|v_{j}\right\|^{2}\right)\right)\right| \\
& =\left|\frac{1}{2} v_{j}^{\mathrm{T}}\left(F_{k}^{\prime \mathrm{T}} F_{k}^{\prime}-\nabla^{2} f\left(x_{k}\right)\right) v_{j}-o\left(\left\|v_{j}\right\|^{2}\right)\right| \\
& =o\left(\left\|v_{j}\right\|^{2}\right) .
\end{aligned}
$$

Using (4.5), we can get 


$$
\begin{aligned}
& f\left(x_{k}\right)-\psi_{k}\left(v_{j}\right)=-g_{k}^{\mathrm{T}} v_{j}-\frac{1}{2} v_{j}^{\mathrm{T}} F_{k}^{\prime \mathrm{T}} F_{k}^{\prime} v_{j} \\
& =\left(-\theta_{j} r_{j}+F_{k}^{\prime \mathrm{T}} F_{k}^{\prime} v_{j}\right)^{\mathrm{T}} v_{j}-\frac{1}{2} v_{j}^{\mathrm{T}} F_{k}^{\prime \mathrm{T}} F_{k}^{\prime} v_{j}\left(\text { because } \theta_{j} r_{j}=\nabla \varphi_{k}\left(v_{j}\right)=g_{k}+F_{k}^{\prime \mathrm{T}} F_{k}^{\prime} v_{j}\right) \\
& =-\theta_{j} r_{j}^{\mathrm{T}} v_{j}+v_{j}^{\mathrm{T}} F_{k}^{\prime \mathrm{T}} F_{k}^{\prime} v_{j}-\frac{1}{2} v_{j}^{\mathrm{T}} F_{k}^{\prime \mathrm{T}} F_{k}^{\prime} v_{j}=-\theta_{j} r_{j}^{\mathrm{T}}\left(\sum_{i=0}^{j-1} \lambda_{i} d_{i}\right)+\frac{1}{2} v_{j}^{\mathrm{T}} F_{k}^{\prime \mathrm{T}} F_{k}^{\prime} v_{j} \\
& =\frac{1}{2} v_{j}^{\mathrm{T}} F_{k}^{\prime \mathrm{T}} F_{k}^{\prime} v_{j} \geqslant \frac{\zeta}{2}\left\|v_{j}\right\|^{2} .
\end{aligned}
$$

Therefore,

$$
\frac{f\left(x_{k}\right)-f\left(x_{k}+v_{j}\right)}{f\left(x_{k}\right)-\psi_{k}\left(v_{j}\right)} \geqslant 1-\frac{o\left(\left\|v_{j}\right\|^{2}\right)}{f\left(x_{k}\right)-\psi_{k}\left(v_{j}\right)} \geqslant 1-\frac{o\left(\left\|v_{j}\right\|^{2}\right)}{\frac{\zeta}{2}\left\|v_{j}\right\|^{2}} \rightarrow 1 .
$$

The above inequality means that there exists $\xi \in(0,1)$ such that

$$
f\left(x_{k}\right)-f\left(x_{k}+v_{j}\right) \geqslant \xi\left[f\left(x_{k}\right)-\psi_{k}\left(v_{j}\right)\right] .
$$

So, each $v_{j}$ generated by step 5 of the algorithm must satisfy (2.3) for sufficiently large $k$, from the algorithm, we can deduce that $p_{k}=-\left(F_{k}^{\prime} \mathrm{T} F_{k}^{\prime}\right)^{-1} g_{k}$.

Next, it will be proved that $p_{k}=-\left(F_{k}^{\prime T} F_{k}^{\prime}\right)^{-1} g_{k}$ satisfies (2.4). Von Neumann Lemma yields $\left(F_{k}^{\prime} F_{k}^{\prime}\right)^{-1}$ is bounded. Combining this result with Theorem 4.1, we can deduce

$$
\lim _{k \rightarrow \infty}\left\|p_{k}\right\|=0
$$

Because $f\left(x_{k}\right)$ is twice continuously differentiable, $g_{k}^{\mathrm{T}} p_{k}=-p_{k}^{\mathrm{T}} F_{k}^{\prime \mathrm{T}} F_{k}^{\prime} p_{k}$, by (4.5), we have that

$$
\begin{aligned}
f\left(x_{k}+p_{k}\right)= & f\left(x_{k}\right)+g_{k}^{\mathrm{T}} p_{k}+\frac{1}{2} p_{k}^{\mathrm{T}} \nabla^{2} f\left(x_{k}\right) p_{k}+o\left(\left\|p_{k}\right\|^{2}\right) \\
= & f\left(x_{k}\right)+\beta g_{k}^{\mathrm{T}} p_{k}+\left(\frac{1}{2}-\beta\right) g_{k}^{\mathrm{T}} p_{k}+\frac{1}{2}\left(g_{k}^{\mathrm{T}} p_{k}+p_{k}^{\mathrm{T}} F_{k}^{\prime \mathrm{T}} F_{k}^{\prime} p_{k}\right) \\
& +\frac{1}{2} p_{k}^{\mathrm{T}}\left[\left(F_{k}^{\prime \mathrm{T}} F_{k}^{\prime}+\sum_{i=1}^{n} \nabla^{2} F_{i}\left(x_{k}\right) F_{i}\left(x_{k}\right)\right)-F_{k}^{\prime \mathrm{T}} F_{k}^{\prime}\right] p_{k}+o\left(\left\|p_{k}\right\|^{2}\right) \\
\leqslant & f\left(x_{k}\right)+\beta g_{k}^{\mathrm{T}} p_{k}-\left(\frac{1}{2}-\beta\right) p_{k}^{\mathrm{T}} F_{k}^{\prime \mathrm{T}} F_{k}^{\prime} p_{k}+o\left(\left\|p_{k}\right\|^{2}\right) \\
\leqslant & f\left(x_{l(k)}\right)+\beta g_{k}^{\mathrm{T}} p_{k}-\left(\frac{1}{2}-\beta\right) \zeta\left\|p_{k}\right\|^{2}+o\left(\left\|p_{k}\right\|^{2}\right) .
\end{aligned}
$$

So, the step size $\alpha_{k}=1$ will be taken for sufficiently large $k$. 
It follows from the above discussions that

$$
x_{k+1}=x_{k}-\left(F_{k}^{\prime} F_{k}^{\prime}\right)^{-1} g_{k},
$$

which implies that for sufficiently large $k$, the step becomes Newton or quasiNewton step, so (4.3) holds.

\section{Numerical Experiments}

In this section, we report some numerical experiments, all codes were written in MATLAB with double precision. In order to check effectiveness of the method, we select the parameters as following: $\varepsilon=10^{-6}, \xi=0.02, \beta=0.4, \omega=0.5$. Our numerical results are listed in Tables $1,2,3$ and 4 . In these tables, $n$ means the number of variables, NF, NG, and NL stand for the number of function evaluations, gradient evaluations, and line search evaluations, respectively, $M$ denotes the nonmonotonic parameter.

In Table 1, we test our proposed algorithm through 6 problems which are quoted from [14] and [13]. The results show that Algorithm NCGL is highly accurate.

In the next experiments, we compare Algorithm NCGL with three-term CG method (MDL) [18], inexact Newton method [6] and Trust region method (NSCTR) [19], respectively. The numerical results are listed in Tables 2, 3 and 4. NOI in Table 2 means the number of iterations, which is equivalent to NG. The computational experiments presented illustrate that in most cases, our algorithm needs fewer iterations. Therefore, we have better results than those reported.

Table 1 Numerical results

\begin{tabular}{|c|c|c|c|c|c|c|c|c|}
\hline \multirow[t]{3}{*}{ Problem } & \multirow[t]{3}{*}{$n$} & \multirow{3}{*}{$\begin{array}{l}\text { Initial } \\
\text { point }\end{array}$} & \multicolumn{6}{|c|}{ NCGL } \\
\hline & & & \multicolumn{3}{|c|}{$M=0$} & \multicolumn{3}{|c|}{$M=3$} \\
\hline & & & NG & NF & $f\left(x_{*}\right)$ & NG & $\mathrm{NF}$ & $f\left(x_{*}\right)$ \\
\hline SC207 & 2 & $(-1.2,1)$ & 7 & 9 & 0 & 5 & 5 & $1.2326 \times 10^{-32}$ \\
\hline SC208 & 2 & $(-1.2,1)$ & 139 & 154 & $1.1359 \times 10^{-28}$ & 145 & 149 & $8.3816 \times 10^{-29}$ \\
\hline $\mathrm{SC} 211$ & 2 & $(-1.2,1)$ & 339 & 358 & $1.6024 \times 10^{-27}$ & 87 & 91 & $5.3841 \times 10^{-28}$ \\
\hline $\begin{array}{l}\text { Powell badly } \\
\text { scaled }\end{array}$ & 2 & $(0,1)$ & 9 & 15 & $1.6713 \times 10^{-4}$ & 13 & 14 & $3.9459 \times 10^{-17}$ \\
\hline $\begin{array}{l}\text { Reklaitis } \\
\text { Ragsdell }\end{array}$ & 2 & $(8,9)$ & 10 & 10 & $2.1457 \times 10^{-28}$ & 10 & 10 & $2.1457 \times 10^{-28}$ \\
\hline $\begin{array}{l}\text { Extended } \\
\text { White \& } \\
\text { Holst }\end{array}$ & 100 & $(-1.2,1, \cdots,-1.2,1)$ & 553 & 564 & $-1.5179 \times 10^{-19}$ & 454 & 456 & $1.8288 \times 10^{-23}$ \\
\hline
\end{tabular}




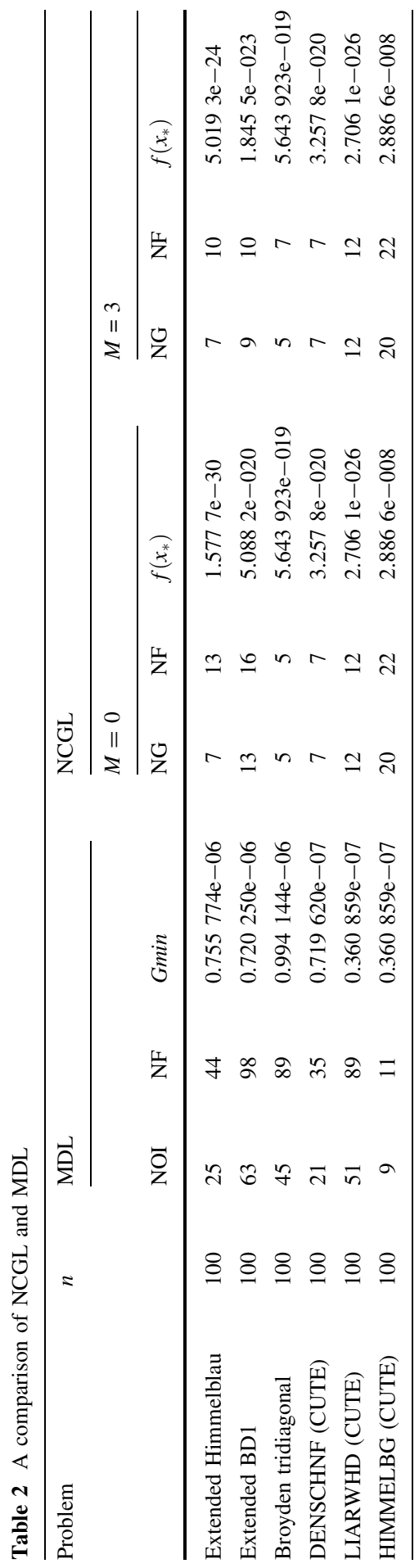




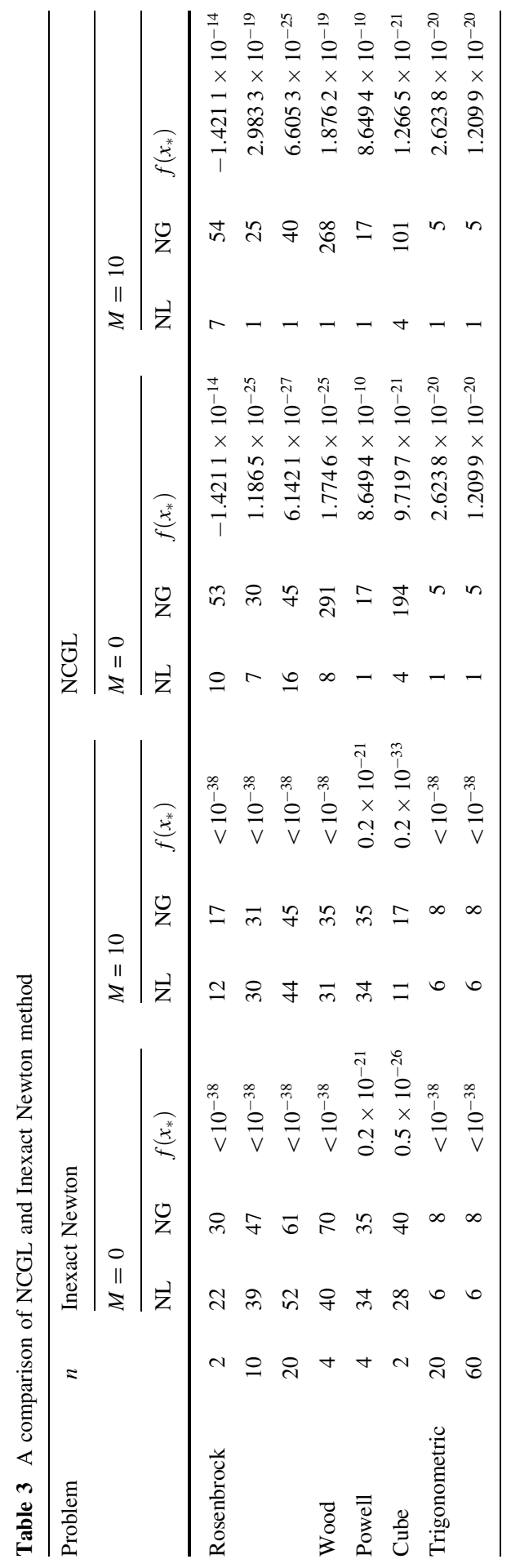


Table 4 A comparison of NSCTR and NCGL

\begin{tabular}{|c|c|c|c|c|c|c|c|}
\hline \multirow[t]{2}{*}{ Problem } & \multirow[t]{2}{*}{$n$} & \multicolumn{3}{|c|}{ NSCTR } & \multicolumn{3}{|c|}{ NCGL $(M=3)$} \\
\hline & & NG & $\mathrm{NF}$ & $f\left(x_{*}\right)$ & NG & $\mathrm{NF}$ & $f\left(x_{*}\right)$ \\
\hline P1 & 100 & 13 & 16 & $5.328164 \mathrm{e}-008$ & 9 & 10 & $5.651849 \mathrm{e}-025$ \\
\hline P3 & 100 & 78 & 85 & $2.949721 \mathrm{e}-007$ & 19 & 19 & $8.437139 \mathrm{e}-010$ \\
\hline P7 & 100 & 112 & 121 & $4.134380 \mathrm{e}-007$ & 42 & 43 & $4.128950 \mathrm{e}-021$ \\
\hline P8 & 100 & 13 & 17 & $1.482496 \mathrm{e}-009$ & 2 & 2 & $8.146554 \mathrm{e}-027$ \\
\hline P9 & 100 & 10 & 11 & $1.544086 \mathrm{e}-005$ & 16 & 16 & $1.359861 \mathrm{e}-009$ \\
\hline
\end{tabular}

Acknowledgments The authors would like to thank the referees for their valuable comments which greatly improved the presentation of this manuscript.

\section{References}

[1] Bulteau, J.P., Vial, JPh: Curvilinear path and trust region in unconstrained optimization, a convergence analysis. Math. Program. Study 30, 82-101 (1987)

[2] Conn, A., Could, N., Toint, P.: Trust-Region Methods. SIAM, Philadelphia (2000)

[3] Dai, Y., Yuan, Y.: A nonlinear conjugate gradient method with a strong global convergence property. SIAM J. Optim. 10(1), 177-182 (1999)

[4] Dai, Y., Kou, C.: A nonlinear conjugate gradient algorithm with an optimal property and an improved wolfe life search. SIAM J. Optim. 23(1), 296-320 (2013)

[5] Gould, N.I.M., Lucidi, S., Roma, M., Toint, P.L.: Solving the trust-region subproblem using the Lanczos method. SIAM J. Optim. 9(2), 504-525 (1999)

[6] Gripp, L., Lampariello, F., Lucidi, S.: A nonmonotone line search technique for Newton's methods. SIAM J. Numer. Anal. 23, 707-716 (1986)

[7] Guan, Y., Zhu, D.: Solving the unconstrained nonlinear optimization using the Lanczos path method. Numer. Math. A J. Chin. Univ. S1, 46-51 (2005)

[8] Guo, P., Zhu, D.: A nonmonotonic interior point algorithm via optimal path for nonlinear optimization with bounds to variables. J. Shanghai Normal Univ. 33(3), 23-29 (2004)

[9] Hager, W., Zhang, H.: A new conjugate gradient method with guaranteed descent and efficient line search. SIAM J. Optim. 16(1), 170-192 (2005)

[10] Hager, W., Zhang, H.: A survey of nonlinear conjugate gradient methods. Pac. J. Optim. 2, 35-58 (2006)

[11] Jia, C., Zhu, D.: An affine scaling interior algorithm via Lanczos path for solving bound-constrained nonlinear systems. Appl. Math. Comput. 195, 558-575 (2008)

[12] Narushima, Y., Yabe, H., Ford, J.: A three-term conjugate gradient method with sufficient descent property and unconstrained optimization. SIAM J. Optim. 21(1), 212-230 (2011)

[13] Neculai, A.: An unconstrained optimization test functions collection. Adv. Model. Optim. 20(1), 147-161 (2008)

[14] Schittkowski, K.: More Test Examples for Nonlinear Programming Codes. Lecture Notes in Economics and Mathematical Systems. Springer, Berlin (1987)

[15] Sun, J.: A convergence proof for an affine-scaling algorithm for convex quadratic programming without nondegeneracy assumptions. Math. Program. 60, 69-79 (1993)

[16] Sun, W., Yuan, Y.: Optimization Theory and Methods. Springer, Berlin (2006)

[17] Ye, Y.: On affine scaling algorithms for nonconvex quadratic programming. Math. Program. 56, 285-300 (1992)

[18] Zhang, J., Xiao, Y., Wei Z.: Nonlinear conjugate gradient methods with sufficient descent condition for large scale unconstrained optimization, Math. Prob. Eng. 16 p (2009). doi:10.1155/2009/243290

[19] Zhou, Q., Zhou, F., Cao, F.: A nonmonotone trust region method based on simple conic models for unconstrained optimization. Appl. Math. Comput. 225, 295-305 (2013)

[20] Zhu, D.: Curvilinear paths and trust region methods with nonmonotonic back tracking technique for unconstrained optimization. J. Comput. Math. 19, 241-258 (2001) 